\title{
The Application of RBF Neural Network Optimized by K-means and Genetic-backpropagation in Fault Diagnosis of Power Transformer
}

\author{
Xinxin $\mathrm{Mi}^{1{ }^{1 *}, \text { Gopinath Subramani }}{ }^{1}$ and Mieowkee Chan ${ }^{1}$ \\ ${ }^{1}$ Centre for Advanced Electrical and Electronic System, Faculty of Engineering, Built Environment, and Information Technology, SEGi \\ University, Jalan Teknologi, Kota Damansara, 47810 Petaling Jaya, Selangor, Malaysia.
}

\begin{abstract}
Through the dissolved gas analysis (DGA) in transformer oil, the fault of the power transformer can be diagnosed. However, the DGA method has the disadvantage of low accuracy because it couldn't exactly reflect the nonlinear relationship between the characteristic gases and fault types. Radial basis function neural network (RBFNN) has the advantage of dealing with complex nonlinear problems, so it can be applied to transformer fault diagnosis based on DGA. The centers, widths and weights has important effects on the performance of the RBFNN. However, it is difficult to find the global optimal solution of these parameters when RBFNN training. This paper creatively designs a method to improve these parameters of RBFNN, firstly using the K-means algorithm to optimize the centers and widths of RBFNN, then using the genetic algorithm-backpropagation (GA-BP) algorithm optimize the weights. Finally, establish the K-means RBF-genetic backpropagation (KRBF-GBP) algorithm model through a large amount of training data. The test results show that the fault diagnosis accuracy of the KRBF-GBP algorithm is $96.4 \%$, higher than the unoptimized RBFNN with $71.43 \%$.
\end{abstract}

\section{Introduction}

Power transformers are an essential part of the power system [1]. Dissolved gas analysis (DGA) is a method of transformer fault diagnosis by analyzing the composition and content of dissolved gas in transformer oil. Compare with the common outage maintenance of power equipment, DGA method will not damage the insulation of the equipment, and it has the advantages of simplicity and convenience [2]. So this method is one of the most effective methods to judge the faults of oil-immersed power transformers [3].

DGA method is used to judge the fault type by known dissolved gas content. However, the corresponding relationship between dissolved gas content and fault type is complex, which is nonlinear, and DGA method does not fully reflect this relationship between them [4]. Therefore, the accuracy of fault diagnosis use DGA method is low. For example, the diagnostic accuracy of the Key gas method which is one of DGA method is only 43.34\% [5]. Radial basis function neural network (RBFNN) is an excellent forward network [6], it has strong advantages in processing complex nonlinear mapping problems, the hidden layer transforms the input vector from the low dimensional space into the high dimensional space, then the linear inseparability problem which in the low dimensional space is linear separable in the high dimensional space [7]. So it can realize the classification of fault very well and could improve the accuracy when applying it in transformer fault diagnosis based on DGA.

The centers, widths and weights are the main parameters of RBFNN, it has important effects on the performance of the RBFNN. However, it is difficult to find the global optimal solution of them [7]. Therefore, how to find the optimal solution of these parameters or optimize them is a worthwhile research topic.

This paper proposes a strategy to optimize the parameters of RBFNN based on DGA data that by using the K-means and the genetic algorithm-backpropagation (GA-BP) algorithm optimizes the parameters of the RBFNN, finally establish the K-means RBF-genetic backpropagation (KRBF-GBP) algorithm model. It can be seen from the test result that this model has a high fault diagnosis rate in transformers and has great application value.

\section{Dissolved gas analysis}

\subsection{Normal value of characteristic gases}

A comparison with the normal value of the dissolved gas in oil can determine the presence or absence of fault. If any of the hydrocarbon gases exceed the contents listed

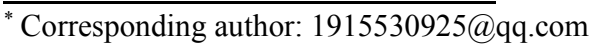


in Table 1, the transformer is considered to be running abnormally [8].

Table 1. Normal value of dissolved gases in oil [8]

\begin{tabular}{cccccc}
\hline Gas components & $\mathbf{H}_{2}$ & $\mathbf{C H}_{4}$ & $\mathbf{C}_{2} \mathbf{H}_{\mathbf{4}}$ & $\mathbf{C}_{2} \mathbf{H}_{\mathbf{6}}$ & $\mathbf{C}_{\mathbf{2}} \mathbf{H}_{\mathbf{2}}$ \\
\hline $\begin{array}{c}\text { Normal limit } \\
(\mu \mathrm{L} / \mathrm{L})\end{array}$ & 100 & 45 & 55 & 35 & 5 \\
& & & & &
\end{tabular}

\subsection{Duval triangle method}

Duval triangle method was proposed by Michelle Duval [9], and it is recommended in the latest IEEE and China National Standard Guide [10].

The Duval triangle method is based on the use of three gases $\mathrm{CH}_{4}, \mathrm{C}_{2} \mathrm{H}_{4}$, and $\mathrm{C}_{2} \mathrm{H}_{2}$ and their location in a triangular map [11]. For plotting the triangle, gases are transformed into triangular co-ordinates [12]. Thermal fault $\left(\mathrm{t}<300^{\circ} \mathrm{C}\right)(\mathrm{T} 1)$, thermal fault $\left(300^{\circ} \mathrm{C}<\mathrm{t}<700^{\circ} \mathrm{C}\right)$ (T2), thermal fault $\left(\mathrm{t}>700^{\circ} \mathrm{C}\right)$ (T3), low energy discharge (D1), high energy discharge (D2), partial discharge (PD), and a mixed region $(\mathrm{D}+\mathrm{T})$ are the seven detectable fault types.

The first step in applying the Duval triangle method is to calculate the percentage of the three gases and then find out the corresponding fault region in Fig. 1. The calculation method of the percentage of the three gases as follows.

$\% \mathrm{CH}_{4}=100 \mathrm{Z} / \mathrm{X}+\mathrm{Y}+\mathrm{Z}, \mathrm{Z}=\left[\mathrm{CH}_{4}\right]$, unit: $\mathrm{uL} / \mathrm{L}$.

$\% \mathrm{C}_{2} \mathrm{H}_{4}=100 \mathrm{Y} / \mathrm{X}+\mathrm{Y}+\mathrm{Z}, \mathrm{Y}=\left[\mathrm{C}_{2} \mathrm{H}_{4}\right]$, unit: $\mathrm{uL} / \mathrm{L}$.

$\% \mathrm{C}_{2} \mathrm{H}_{2}=100 \mathrm{X} / \mathrm{X}+\mathrm{Y}+\mathrm{Z}, \mathrm{X}=\left[\mathrm{C}_{2} \mathrm{H}_{2}\right]$, unit: uL / L.

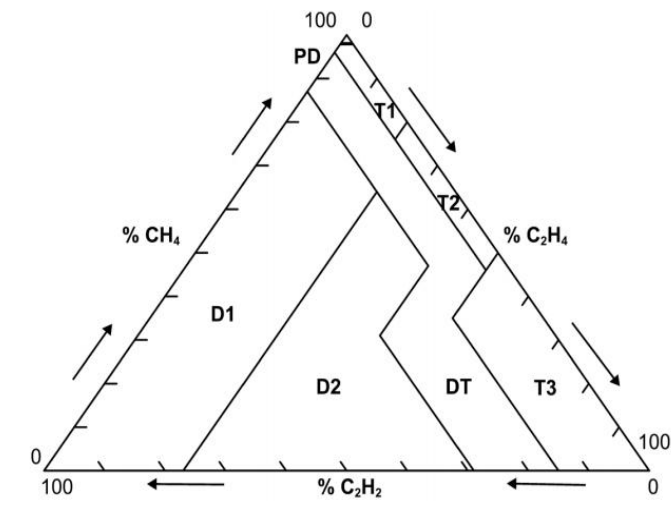

Fig. 1. Duval triangle method [13]

Table 2. Fault zone boundaries for Fig. 1 [13]

\begin{tabular}{cccc}
\hline $\begin{array}{c}\text { Gas (\%) } \\
\text { / Fault }\end{array}$ & $\% \mathrm{CH}_{4}$ & $\% \mathrm{C}_{2} \mathrm{H}_{4}$ & $\% \mathbf{C}_{2} \mathrm{H}_{2}$ \\
\hline PD & $\geq 98$ & - & - \\
\hdashline T1 & $<98$ & $<20$ & $<4$ \\
\hdashline T2 & - & $\geq 20$ and $<50$ & $<4$ \\
\hdashline T3 & - & $\geq 50$ & $<15$ \\
& - & $<50$ & $\geq 4$ and $<13$ \\
DT & - & $\geq 40$ and $<50$ & $\geq 13$ and $<29$ \\
\hline
\end{tabular}

\begin{tabular}{cccc}
\hline & - & $\geq 50$ & $\geq 15$ and $<29$ \\
\hline D1 & - & $<23$ & $\geq 13$ \\
& - & $\geq 23$ & $\geq 29$ \\
D2 & - & $\geq 23$ and $<40$ & $\geq 13$ and $<29$ \\
\hline
\end{tabular}

\subsection{Rogers ratios method}

This method uses three gas ratios involving five gas concentrations, which are $\mathrm{C}_{2} \mathrm{H}_{2} / \mathrm{C}_{2} \mathrm{H}_{4}, \mathrm{CH}_{4} / \mathrm{H}_{2}$, and $\mathrm{C}_{2} \mathrm{H}_{4} / \mathrm{C}_{2} \mathrm{H}_{6}$. The range of each gas ratio suggesting a particular fault type is given in Table 3 [14].

Table 3. Rogers ratios method [13]

\begin{tabular}{|c|c|c|c|c|}
\hline $\begin{array}{l}\text { Ca } \\
\text { se }\end{array}$ & $\begin{array}{l}\mathrm{C}_{2} \mathbf{H}_{2} / \\
\mathrm{C}_{2} \mathbf{H}_{4} \\
\end{array}$ & $\mathrm{CH}_{4} / \mathrm{H}_{2}$ & $\begin{array}{l}\mathrm{C}_{2} \mathbf{H}_{4} / \\
\mathrm{C}_{2} \mathbf{H}_{6} \\
\end{array}$ & $\begin{array}{c}\text { Suggested fault } \\
\text { diagnosis }\end{array}$ \\
\hline 0 & $<0.1$ & 0.1 to 1.0 & $<1.0$ & Unit normal \\
\hline 1 & $<0.1$ & $<0.1$ & $<1.0$ & $\begin{array}{l}\text { Low-energy } \\
\text { density } \\
\text { arcing-PD }\end{array}$ \\
\hline 2 & 0.1 to 3.0 & 0.1 to 1.0 & $>3.0$ & $\begin{array}{l}\text { Arcing-High-en } \\
\text { ergy discharge }\end{array}$ \\
\hline 3 & $<0.1$ & 0.1 to 1.0 & 0.1 to 3.0 & $\begin{array}{c}\text { Low temperature } \\
\text { thermal }\end{array}$ \\
\hline 4 & $<0.1$ & $>1.0$ & 0.1 to 3.0 & Thermal $<700^{\circ} \mathrm{C}$ \\
\hline 5 & $<0.1$ & $>1.0$ & $>3.0$ & Thermal $>700^{\circ} \mathrm{C}$ \\
\hline
\end{tabular}

\section{RBF neural network and K-means}

\subsection{RBF neural network}

RBFNN is an efficient feed-forward neural network, which has the best approximation performance and global optimal characteristics, and it's simple in structure and fast in training. Therefore, the RBFNN model can be widely used in pattern recognition, nonlinear function approximation, and other fields [15].

RBFNN contains the input layer, the hidden layer, the output layer. The structure is shown in Fig. 2.

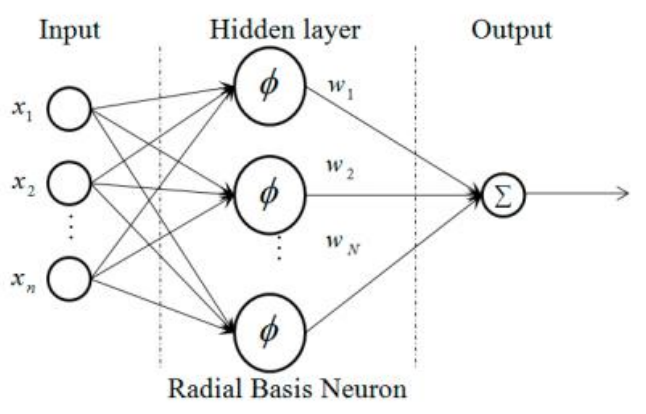

Fig. 2. Structure of the RBFNN [16]

In this paper, the Gaussian function is selected as the function of the hidden layer, the calculate method as shown in formula (1). 


$$
\phi_{i}(x)=\exp \left(-\frac{\left\|x-x_{c}\right\|^{2}}{2 \sigma^{2}}\right)
$$

Where $\phi_{i}$ is the output of the hidden layer, $x$ is the input value of the RBFNN, $x_{c}$ are the centers of the Gaussian function, $\sigma$ are the widths of the Gaussian function.

The outputs of the RBFNN is shown in formula (2).

$$
y=f(x)=\sum_{i}^{N} w_{i} \phi_{i}(x)
$$

Where $f(x)$ is the output value of the RBFNN and $w_{i}$ are the weights between the hidden layer and the output layer. The output value of the RBFNN is the sum of products of $\phi_{i}$ and $w_{i} . x_{c}, \sigma$ and $w$ are important indicators to evaluate the performance of RBFNN.

\subsection{K-means clustering algorithm (KMCA)}

$\mathrm{K}$-means algorithm is a kind of unsupervised learning algorithm that is often used in clustering analysis. Because of its simple working steps, high efficiency, it is widely used in the task of data mining and pattern recognition [17].

The principle of K-means algorithm is that for the input sample sets, data points are randomly generated as the initial centers, the euclidean distance between each sample point and each center is calculated, and each sample point is divided into the nearest cluster where the centers are located, the centers are recalculated, and the position of the sample points are updated iteratively continuously until the centers no longer change.

K-means algorithm can be used to adjust the clustering centers in the selection of the RBFNN centers to make the selection of the network centers more accurate [18].

\section{BP neural network and genetic algorithm}

\subsection{BP neural network}

BPNN is a multi-layer network, it generally consists of an input layer, one or more hidden layers, and an output layer. Adjust the weights and thresholds by propagating the error between the expected output and the actual output, achieve the training of BPNN [19]. Forward propagation of signals and back-propagation of errors is a complete learning process of the BPNN [20]. Fig. 3 shows a three-layer BPNN structure with a hidden layer.

Where, there are $\mathrm{n}$ neurons in the input layer, $\mathrm{h}$ neurons in the hidden layer and $\mathrm{m}$ neurons in the output layer. $w_{i j}$ is the connection weight between the jth neuron of the input layer $x_{j}$ and the ith neuron of the hidden layer, $b_{i}$ is the threshold of the ith neuron of the hidden layer. $w_{k i}$ is the connection weight between the ith neuron of the hidden layer and the kth neuron of the output layer $y_{k}, a_{k}$ is the threshold of $y_{k}$. Besides, $\varphi$ represents the activation function of the hidden layer and $\psi$ represents the activation function of the output layer [21].

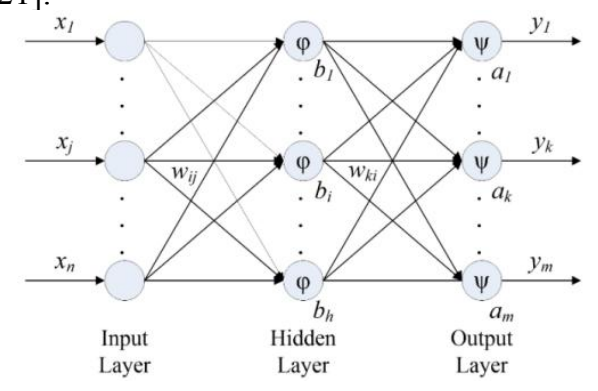

Fig. 3. The structure of the BPNN [21]

\subsection{Genetic algorithm (GA)}

Genetic algorithm (GA) is a kind of random optimization search algorithm that draws on the natural selection and genetic principle of the biological community. Its main characteristic is the group search strategy and the information exchange among the individuals in the group, the search does not depend on the gradient information [22].

The GA is different from the traditional search algorithm, which is based on a fitness function and realizes the iterative process search of individual structure reorganization in the population by carrying out the genetic operation on all individuals in the population. Selection, crossover, and mutation constitute three main genetic operations of the GA. Parameter coding, initial population setting, fitness function design, genetic operation design, control parameter setting, and other elements constitute the core content of the GA [22].

\subsection{GA-BP algorithm}

Because the BP algorithm is very sensitive to the initial weights and thresholds, different initial weights and thresholds may lead to different results and easily fall into local minimums and other problems [24]. So it can be compensated for the weak global search ability of the BPNN by the superior global search ability of GA [25]. The algorithm of optimizing BPNN by GA is called the GA-BP algorithm. The initial weights and thresholds of BPNN are optimized by GA to improve the learning convergence speed of the neural network so that the optimized BPNN can better predict the output of function [26].

The description of GA-BP algorithm.

Step 1. Input the sample data, and determine the structure of the BPNN (node numbers of the input layer, node numbers of the hidden layer, node numbers of output layer).

Step 2. GA (fitness evaluation, selection, crossover, mutation) is used to optimize the weights and thresholds of BPNN. The optimal individual is obtained. 
Step 3. The optimal individual are taken as the initial weights and thresholds of the BPNN, and then trained it by the BP algorithm.

Step 4. Gets the error between the actual outputs and the expected outputs of the BP network. Determine whether the error meets the accuracy requirements. If meet the requirements, the algorithm ends; if not meet the requirements, continue to $\mathrm{BP}$ algorithm training.

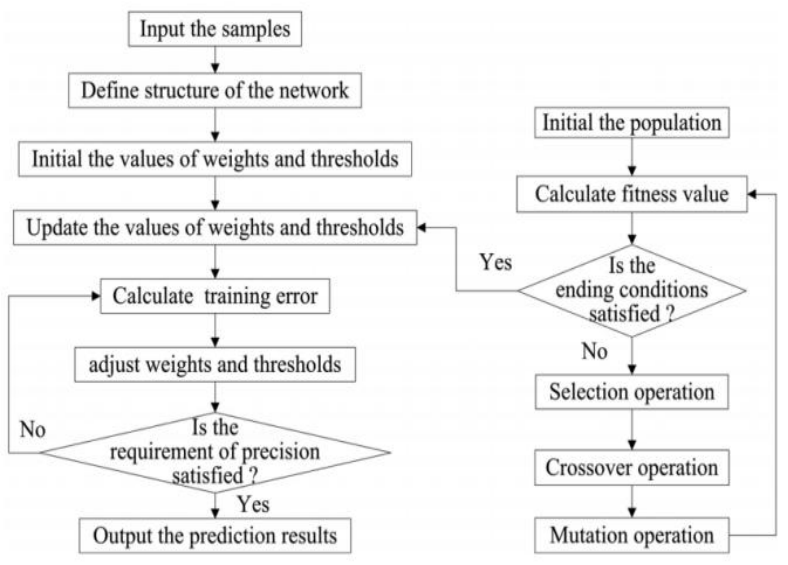

Fig. 4. The operation process of the GA-BP algorithm [27]

\section{Methodology}

\subsection{Research design}

This paper uses both quantitative and qualitative research methods.

The quantitative method is used in three aspects, which are, the DGA data analysis; the parameter setting of RBFNN, BPNN, and GA; and the calculation of the accuracy of the improved RBFNN algorithm.

The qualitative method is used in the classification of transformer fault types (T1, T2, T3, D1, D2, PD). There are many classification methods in this paper, which are, Duval triangle method, Rogers ratios method, and newly established the KRBF-GBP algorithm.

\subsection{Research data}

\subsubsection{Data collection}

The research data are collected from journal papers and dissertations related to the research. A total of 364 sets (31 sets for T1, 54 sets for T2, 125 sets for T3, 54 sets for D1, 80 sets for D2, 20 sets for PD) of DGA data as training sets, a total of 110 sets ( 15 sets for T1, 18 sets for T2, 31 sets for T3, 14 sets for D1, 20 sets for D2, 12 sets for PD) of DGA data as testing sets.

\subsubsection{Data analysis}

Step1. Before network training and testing, the collected data must be verified. As shown in Table 1, if the concentration of a set of data is all below the normal limit, then delete it. If the concentration of any gas is higher than the normal limit, then this set of data can be used for step 2.

Step 2. This research uses the Duval triangle method [13], Rogers ratios method [13], and the actual faults given by journals to verify the DGA data. There will be three diagnostic results, if two or three of them are the same, the transformer is considered to belong to this type of fault.

\section{The design of improve RBFNN structure}

From the view of network structure, between the hidden layer and the output layer of RBFNN is weight connection. BPNN uses weight connection too [23]. Therefore the optimization problem of weights between the hidden layer and the output layer of RBFNN can be solved by optimizing the parameters (weights and thresholds) of the BPNN model.

The transfer process as shown in the dotted lines of Fig. 5, the network between the hidden layer and the output layer of the RBFNN is changed into a three-layer BPNN.

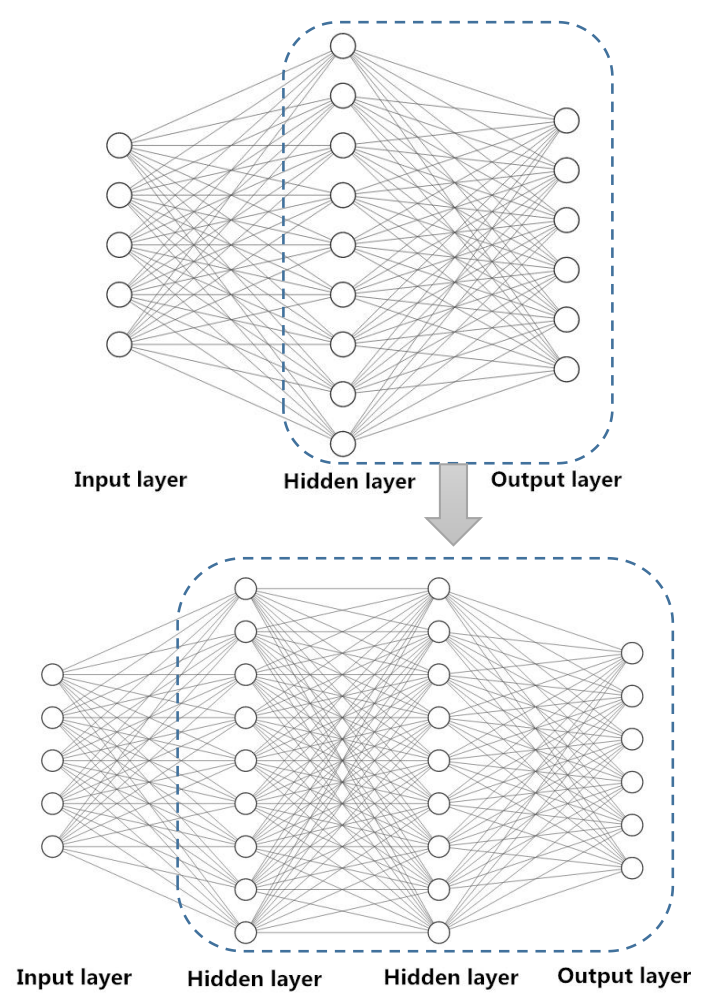

Fig. 5. The chart of RBFNN structure change

Note: The number of network nodes in Fig. 5 is randomly determined. It just helps the author explain more easily.

Where, the hidden layer and output layer of the RBFNN are circled with the first dotted lines, the BPNN is circled with the second dotted lines.

It also can know from the sub-section 'GA-BP Algorithm', GA realizes the optimization of the initial weights and thresholds of BPNN. Therefore, the 
optimization process of the weights of RBFNN is realized by the GA-BP algorithm.

\section{Training and testing of the RBF algorithm}

The process of RBFNN training and testing is shown in Fig. 6.

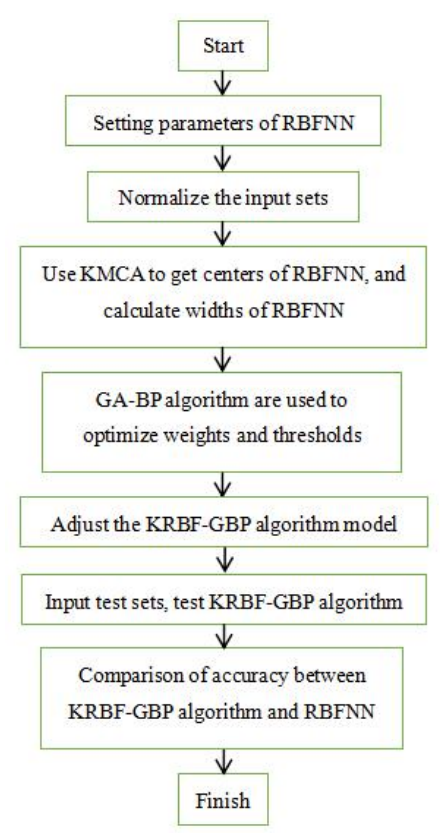

Fig. 6. The process of RBFNN training and testing

Step 1. Setting parameters of RBFNN (inputs, outputs, and nodes of RBFNN).

Step 2. Normalization of the input sets.

Step 3. Running the K-means clustering algorithm (KMCA), get the centers of RBFNN, and calculate the widths of RBFNN.

Step 4. Using the GA-BP algorithm to optimize the weights and thresholds of BPNN (choice of parameters of BPNN; setting parameters of GA; choice of epochs of BPNN).

Step 5. The adjustment of the KRBF-GBP algorithm model.

Step 6. Inputting test set, test the KRBF-GBP algorithm, and calculate the accuracy. Finally, compare the accuracy of the KRBF-GBP algorithm with the unimproved RBFNN.

The following contents will introduce to the process of RBFNN training and testing in detail.

\subsection{The training of RBFNN}

\subsubsection{Setting inputs, outputs, and nodes of RBFNN}

Given that the number of input nodes of RBFNN is $5\left(\mathrm{H}_{2}\right.$, $\mathrm{CH}_{4}, \mathrm{C}_{2} \mathrm{H}_{4}, \mathrm{C}_{2} \mathrm{H}_{6}, \mathrm{C}_{2} \mathrm{H}_{2}$ ).

The outputs of the model are the fault types of transformer, which are, low temperature overheating (T1), medium temperature overheating (T2), high temperature overheating (T3), low energy discharge (D1), high energy discharge (D2), partial discharge (PD).

Table 4. The fault types and codes of expected output

\begin{tabular}{ccccccc}
\hline Fault types & \multicolumn{5}{c}{ Codes of expected output } \\
\hline T1 & 1 & 0 & 0 & 0 & 0 & 0 \\
T2 & 0 & 1 & 0 & 0 & 0 & 0 \\
T3 & 0 & 0 & 1 & 0 & 0 & 0 \\
D1 & 0 & 0 & 0 & 1 & 0 & 0 \\
D2 & 0 & 0 & 0 & 0 & 1 & 0 \\
PD & 0 & 0 & 0 & 0 & 0 & 1 \\
\hline
\end{tabular}

The number of nodes in the input layer, the hidden layer, and the output layer of the 3-layer forward neural network are $n_{i}, n_{h 1}$ and $n_{o}$. In the design of hidden layer nodes, many authors adopt the following formula [28].

$$
n_{h 1}=\sqrt{n_{i}+n_{o}}+m
$$

Where, $m$ is a constant, $m \in[1,10]$.

In this research, $n_{i}=5, n_{o}=6$, so $n_{h 1}$ can be calculated.

$$
n_{h 1}=\sqrt{5+6}+m, m \in[1,10]
$$

So $n_{h 1} \in[4,13]$.

In order to continue the following training, the intermediate value of $n_{h 1}$ is taken, so assume $n_{h 1}$ is 9 .

\subsubsection{Normalization of the input sets}

DGA data for fault diagnosis come from transformers with different capacity and voltage levels, and the volume of gas of the same fault type in the sample is different. For maintaining the identity of the input vectors, the components of input gases need to be normalized, mapping each input vector into an interval of $0 \sim 1$ [29].

\subsubsection{Running the K-means clustering algorithm (KMCA)}

Programming implementation algorithm. Running the code of the K-means algorithm in MATLAB 2016a to get the centers of the hidden layer of RBFNN and calculate the widths of the hidden layer of RBFNN. The outputs of the hidden layer of RBFNN is calculated using the Gaussian function.

\subsubsection{Choice of parameters of BPNN}

The inputs of the BPNN in this research are the outputs of the hidden layer of the RBFNN. Tan sigmoid transfer function is selected as the transfer function of the hidden layer of the BPNN, the pure linear transfer function is selected as the transfer function of the output layer of the BPNN. The Levenberg-Marquardt algorithm is selected 
in the training algorithm of weights because it has the advantages of both the gradient method and the Newton method.

It can be known from sub-section 'Setting inputs, outputs, and nodes of RBFNN', the output node numbers of the hidden layer of RBFNN is $n_{h 1}$, so the input node numbers of BPNN is $n_{h 1}$, the output node numbers is 6 (T1, T2, T3, D1, D2, PD), the node numbers of hidden layer of BPNN is $n_{h 2}$. According to formula (3) and (4).

$$
\begin{gathered}
n_{h 2}=\sqrt{n_{h 1}+6}+m, m \in[1,10], \quad n_{h 1} \in[4,13], \text { so } \\
\sqrt{4+6}+m \leq n_{h 2} \leq \sqrt{13+6}+m, m \in[1,10] .
\end{gathered}
$$

So $n_{h 2} \in[4,14]$.

In order to continue the following training, the intermediate value of $n_{h 2}$ is taken, so assume $n_{h 2}$ is 9 .

\subsubsection{Setting parameters of $G A$}

At present, the selection of GA parameters is based on experience and the simulation test of specific problems[30]. The choice of GA parameters in this paper is also based on the experience of previous simulation tests. The population size of GA is set to 20 , genetic algebra is set to 15 , crossover probability is set to 0.6 , mutation probability is set to 0.05 .

\subsubsection{Choice of epochs of BPNN}

Generally speaking, the smaller the allowable error of the neural network, the higher the fitting degree (R), its prediction accuracy (The determination coefficient $\left(\mathrm{R}^{2}\right)$ is used in this paper) is also higher. However, the practical application shows that the prediction error decreases at the beginning with the decrease of the fitting error (The mean square error (MSE) is used in this paper), but with the decrease of the fitting error to a certain value, the prediction error increases, which indicates that the generalization ability of the network is reduced and the over-fitting occurs [31]. Under-fitting refers to the low fitting degree of the model. Usually, its performance in the training set is poor, and the performance in the test set is also poor. Adjusting the number of epochs of the BPNN can effectively prevent the occurrence of over-fitting and under-fitting.

Running the code of the GA-BP algorithm in MATLAB 2016a for computer simulation, record the variation relationship between $M S E, R$, and $R^{2}$ with epochs, find the best epochs of the network.
Table 5. The relationship between MSE, R, and $R^{2}$ with epochs of BPNN

\begin{tabular}{cccc}
\hline Epochs of BPNN & MSE & $\mathbf{R}$ & $\mathbf{R}^{2}$ \\
\hline 5 & 0.0416 & 0.8374 & 0.8981 \\
10 & 0.0351 & 0.8645 & 0.9121 \\
15 & 0.0314 & 0.88 & 0.9359 \\
20 & 0.0278 & 0.8944 & 0.9485 \\
25 & 0.027 & 0.8972 & 0.9239 \\
30 & 0.0176 & 0.9346 & 0.8829 \\
35 & 0.0186 & 0.9306 & 0.9406 \\
40 & 0.0201 & 0.9246 & 0.9183 \\
50 & 0.0147 & 0.9458 & 0.9116 \\
60 & 0.0132 & 0.9511 & 0.8827 \\
75 & 0.0094 & 0.9656 & 0.6522 \\
120 & 0.0075 & 0.9728 & 0.7656 \\
300 & 0.0059 & 0.9783 & 0.4208 \\
500 & 0.0048 & 0.9826 & 0.2924 \\
\hline
\end{tabular}

It can be known from Table 5 that when the value of epochs is 35 , the prediction accuracy is $94.06 \%$, and the fitting degree is $93.06 \%$. It shows that the network has neither over-fitting nor under-fitting, and the accuracy meets the requirements. Meanwhile, the value of the MSE is 0.0186. So the value of the epochs is set 35 in BPNN.

Running the GA-BP algorithm that sets all the parameters, and get the KRBF-GBP algorithm model.

\subsection{The adjustment of the KRBF-GBP algorithm}

The number of RBFNN hidden layer and the number of BPNN hidden layer, and the relationship between them have a great influence on the performance of KRBF-GBP model.

From sub-section 'Setting inputs, outputs, and nodes of RBFNN' and 'Choice of parameters of BPNN', it can be known that $n_{h 1} \in[4,13], n_{h 2} \in[4,14]$. In this part, the value of $n_{h 1}, n_{h 2}$ are adjusted to get the maximum of the determination coefficient $\mathrm{R}^{2}$.

Running each case three times, then take their average, the results as shown in Table 6.

Table 6. The relationship between the value of $n_{h 1}, n_{h 2}$ and $\mathrm{R}^{2}$

\begin{tabular}{lccccccccccc}
\hline \multicolumn{10}{c}{ The determination coefficient $\left(\mathbf{R}^{2}\right)$} \\
\hline & $n_{h 1}$ & 4 & 5 & 6 & 7 & 8 & 9 & 10 & 11 & 12 & 13 \\
$n_{h 2}$ & & & & & & & & & & & \\
4 & 0.8690 & 0.8430 & 0.8309 & 0.8696 & 0.8840 & 0.9095 & 0.8424 & 0.7926 & 0.9442 & 0.8730 \\
\hline
\end{tabular}




\begin{tabular}{ccccccccccc}
\hline 5 & 0.8931 & 0.8863 & 0.8698 & 0.8661 & 0.8966 & 0.8873 & 0.8767 & 0.9451 & 0.9145 & 0.8776 \\
6 & 0.8948 & 0.8670 & 0.8932 & 0.8839 & 0.8811 & 0.9262 & 0.8895 & 0.9303 & 0.9134 & 0.8963 \\
7 & 0.8689 & 0.8806 & 0.8445 & 0.8908 & 0.9103 & 0.9229 & 0.9410 & 0.8965 & 0.9098 & 0.9246 \\
8 & 0.8892 & 0.9006 & 0.9149 & 0.9276 & 0.9260 & 0.9264 & 0.9157 & 0.8861 & 0.9447 & 0.9129 \\
9 & 0.8826 & 0.8862 & 0.9170 & 0.9083 & 0.9151 & 0.8960 & 0.9442 & 0.9069 & 0.9047 & 0.9205 \\
10 & 0.9006 & 0.9101 & 0.9029 & 0.9330 & 0.9158 & 0.8903 & 0.9258 & 0.9298 & 0.8935 & 0.8838 \\
11 & 0.8745 & 0.8567 & 0.9038 & 0.8930 & 0.9047 & 0.8974 & 0.9344 & 0.9362 & 0.9383 & 0.9292 \\
12 & 0.8760 & 0.9269 & 0.8931 & 0.8898 & 0.9286 & 0.9188 & 0.9319 & 0.9090 & 0.9415 & 0.9147 \\
13 & 0.9048 & 0.9201 & 0.8718 & 0.9146 & 0.9227 & 0.9213 & 0.9217 & 0.8977 & 0.9163 & 0.9246 \\
14 & 0.8627 & 0.8852 & 0.8946 & 0.8846 & 0.8874 & 0.9039 & 0.9412 & 0.9184 & 0.8024 & 0.8960 \\
\hline
\end{tabular}

It can be known from Table 6 , when $n_{h 1}$ is 11 and $n_{h 2}$ is 5 , $\mathrm{R}^{2}$ is the largest, which is 0.9451 . So the number of hidden layers of RBFNN set to 11, the number of hidden layers of BPNN set to 5 .

\subsection{The testing of KRBF-GBP algorithm}

Test all data of the test set, the results as shown in Table 7.

Table 7. The accuracy of the KRBF-GBP algorithm

\begin{tabular}{|c|c|c|c|c|c|c|c|}
\hline \multirow{2}{*}{$\begin{array}{c}\begin{array}{c}\text { Fault } \\
\text { types }\end{array} \\
\text { Expected } \\
\text { outputs }\end{array}$} & \multicolumn{6}{|c|}{ Test outputs } & \multirow{2}{*}{$\begin{array}{c}\text { Accuracy } \\
\text { of } \\
\text { KRBF-GB } \\
\text { P(\%) }\end{array}$} \\
\hline & $\mathrm{T} 1$ & $\mathrm{~T} 2$ & $\mathrm{~T} 3$ & D1 & D2 & PD & \\
\hline $\mathrm{T} 1$ & 15 & 0 & 0 & 0 & 0 & 0 & 100 \\
\hline $\mathrm{T} 2$ & 1 & 16 & 0 & 0 & 0 & 1 & 88.89 \\
\hline $\mathrm{T} 3$ & 1 & 0 & 30 & 0 & 0 & 0 & 96.77 \\
\hline D1 & 0 & 0 & 0 & 13 & 1 & 0 & 92.86 \\
\hline D2 & 0 & 0 & 0 & 0 & 20 & 0 & 100 \\
\hline PD & 0 & 0 & 0 & 0 & 0 & 12 & 100 \\
\hline
\end{tabular}

From Table 7, it can be seen that 15 sets data of fault $\mathrm{T} 1$ are all correct, so its accuracy is $100 \%$. There are 16 sets data of fault T2 are correct, and 2 sets data is misdiagnosed to $\mathrm{T} 1$ and $\mathrm{PD}$, so the accuracy is $88.89 \%$. There are 30 sets data of fault T3 are correct, and 1 set data is misdiagnosed to $\mathrm{T} 1$, so the accuracy is $96.77 \%$. There are 13 sets data of fault D1 are correct, and 1 set data is misdiagnosed to D2, so the accuracy is $92.86 \%$. 20 sets test data of fault D2 are all correct, so its accuracy is $100 \%$. 12 sets test data of fault PD are all correct, so its accuracy is $100 \%$. Finally, it can be known the accuracy of KRBF-GBP algorithm for transformer fault is $96.4 \%$, which is calculated by $(100 \%+88.89 \%+$ $96.77 \%+92.86 \%+100 \%+100 \%) / 6$.

However, the prediction accuracy of the power transformer fault by RBFNN without parameter optimization is only $71.43 \%$ [32]. So the KRBF-GBP algorithm improves the accuracy of power transformer fault diagnosis.

\section{Conclusion}

An optimization scheme of RBFNN parameters is proposed, and the KRBF-GBP algorithm model is established. It can be seen from the test result that the KRBF-GBP algorithm improves the accuracy of the transformer fault diagnosis. Therefore, it can be proved that the scheme of optimizing the parameters of RBFNN by the K-means and GA-BP algorithm is feasible, and the KRBF-GBP algorithm is suitable for transformer fault diagnosis and has great application prospect.

\section{Future scope}

It is undeniable that this research has limitations. Many adjustable variables are involved in the training of the neural network model, such as the nodes numbers of the hidden layer of RBFNN, the iteration numbers of BPNN, the population size of GA, and so on. There are no great methods about how to choose them, and the most widely used is by trial and error method. In this research, many adjustable variables are selected by the hypothesis firstly and then solved by trial and error method. So theoretically, the RBFNN trained by K-means clustering and GA-BP algorithm is difficult to achieve the best training effect, and there is still a better model than it. Hope the problem can be solved in future research works.

Facilities and support provided by SEGi University is highly acknowledged.

\section{References}

1. A.K.Mehta, R.N.Sharma, S.Chauhan, and S.Saho. (2013). Transformer diagnostics under dissolved gases analysis using support vector machine. 2013 International Conference on Power, Energy and Control, 181-186.

2. Wu, Y., Dong, X.Y., and Zhang, C. (2017). Discussion on fault diagnosis technology of transformer based on the dissolved gas method in oil. Electrical Engineering, 5: 71-72.

3. Wan, F., and Chen, W.G. The study of transformer oil-dissolved gases detection based on optical-feedback V-shaped cavity-enhanced absorption spectroscopy. Chongqing University, 
2015.

4. Wang, X.X., and Wang, T. (2008). Transformer fault diagnosis based on BP network. East China Electric Power, 36 (2): 112-115.

5. F.A.Al-Baw, M.Z.Ali, and K.Al-Zyoud. Diagnosis of power transformer depending on the dissolved gas analysis. Department of Electrical Engineering, Al-Balqa' Applied University, 2017.

6. Department of Mathematics, Tongji University. (2007). Engineering mathematics: linear algebra. Beijing: Higher Education Press.

7. Yan, K., Zhao, H., Xu, Q.W., Wang, F.C., Shen, J., and Jiang, X.J. (2015). Identification of insulator hydrophobicity based on image processing and RBF neural network. High Voltage Apparatus, 51 (1): 30-35.

8. Zhang, L.G. (2000). Relationship between the composition and content of dissolved gas in transformer oil and fault diagnosis of insulation of oil-immersed power equipment. Transformer, 37 (3): $39-42$.

9. A.Kbari, A.Setayeshmehr, and H.Borsi. A software implementation of the Duval triangle method. Institute of Electric Power Systems, High Voltage Engineering Section, Germany: Leibniz Universitat, 2015.

10. General Administration of Quality Supervision, Inspection and Quarantine of the People's Republic of China. Guide for analysis and judgment of dissolved gases in transformer oil. GB/T 7252-2001, 2001.

11. R.R.Rogers. (1978). IEEE and IEC codes to interpret incipient faults in transformers using gas in oil analysis. IEEE Transactions on Electrical Insulation, EI-13 (5): 349-354.

12. Huo, C.S., Yann, C.H., and Chao, M.H. (2012). A review of dissolved gas analysis in power transformers. SciVerse ScienceDirect, (14): $1220-1225$.

13. Transformers Committee of the IEEE Power and Energy Society. IEEE guide for the interpretation of gases generated in mineral oil-immersed transformers. IEEE Std C57.104TM-2019, 2019.

14. S.A.Khan, M.D.Equbal, and T.Islam. (2015). A comprehensive comparative study of DGA based transformer fault diagnosis using fuzzy logic and ANFIS models. IEEE Transactions on Dielectrics and Electrical Insulation, 22 (1): 590-596.

15. Kang, W. (2012). Prediction of dissolved gas concentration in transformer oil based on RBF neural network. Science and Technology Innovation and Application, (11): 59-60.

16. He, H.Q., Yan, Y.L., Chen, T., and Cheng, P.G. (2019). Tree height estimation of forest plantation in mountainous terrain from bare-earth points using a DoG-coupled radial basis function neural network. Remote Sensing, (11): 1271.

17. Deng, B.Y. (2020). A review of the K-means optimization algorithm. Computer Engineering \& Software, 41 (2): 188-192.

18. Zhao, L., Jia, Z.H., and Qin, X.Z. (2011). Optimization of RBF network based on K-means and quantum genetic algorithm. Computer Engineering, 37 (10): 152-153.

19. Xu, L.J., and Yang, J.G. (2011). The risk assessment method for money laundering based on a multi-layer neural network. Computer Engineering, 36 (22): 181-183.

20. Qu, Z.Y., Ji, C., Guo, X.L., and Zhang, H. (2014). Improvement of BP network algorithm based on transfer function self-optimization. Electrical Measurement and Instrumentation, 51 (11): 56-64.

21. Sen, W., Liang, M., and Wang, J.F. (2019). Projectile impact point prediction based on genetic algorithm BP neural network. Journal of Physics: Conference Series, 1-8.

22. Frees Technology Product Development Center. (2003). Matlab 6.5 auxiliary optimization calculation and design. Beijing: Electronic Industry Press.

23. Li, J.J. A study on internal fault diagnosis method of transformer based on EBF neural network. Nanjing: Nanjing University of Science \& Technology, 2010.

24. Wu, L.F. (2011). Optimization of BP neural network weights using a cloud model and genetic algorithm. Software Guide, 10 (9): 56-57.

25. Wu, C., and Wang, H.J. (2016). Optimization of BP neural network based on improved adaptive genetic algorithm. Electronic Design Engineering, 24 (24): 29-37.

26. Wang, C.J., Yu, W.D., and Chen, Z.Q. (2003). A BP neural network algorithm is based on genetic algorithm and its application. Journal of Nanjing University (Natural Science Edition), 39 (5): 459-466.

27. Yin, M.H., and Su, H. (2016). Theoretical and experimental studies on dynamics of double-helical gear system supported by journal bearings. Advances in Mechanical Engineering, 8 (5): 1-13.

28. Xia, K.W., Li, C.B., and Shen, J.Y. (2005). An optimization algorithm on the number of hidden layer nodes in the feed-forward neural network. Computer Science, 32 (10): 143-145.

29. Liang, Y.C., and Li, Y.M. (2005). Fault diagnosis of the transformer with improved combined RBF neural network. High Voltage Technology, 31 (9): 31-33.

30. Jin, Q., and Li, X.R. (2006). GA parameter setting and its application in load modeling. Electric Power Automation Equipment, 26 (5): 23-27.

31. Yang, X.L., and Zhou, C.Y. (2014). Over-fitting phenomenon control in BP network forecast. China Science and Technology Papers Online, 1-7.

32. Zheng, W.G., Wang, A.J., Wang, G., and Zhang, X.W. (2019). Research on power transformer fault diagnosis model based on the SA-KM algorithm and RBF neural network. System Modeling, Simulation \& Analysis, 34 (12): 80-91. 